\title{
A liver metastasis 7 years after resection of a low-risk duodenal gastrointestinal stromal tumor
}

\author{
Masashi Inoue ${ }^{1}$, Masayuki Shishida ${ }^{1}$, Atsuhiro Watanabe ${ }^{1}$, Ryujiro Kajikawa ${ }^{1}$, Ryotaro \\ Kajiwara $^{1}$, Hiroyuki Sawada ${ }^{1}$, Ichiro Omori ${ }^{1}$, Kazuaki Miyamoto ${ }^{1}$, Masahiro Ikeda ${ }^{1}$, \\ Kazuhiro Toyota ${ }^{1}$, Seiji Sadamoto ${ }^{1}$, and Tadateru Takahashi ${ }^{1}$ \\ ${ }^{1}$ National Hospital Organization Higashi Hiroshima Medical Center
}

June 11, 2020

\begin{abstract}
Duodenal gastrointestinal stromal tumors (dGISTs) are rare, and a lack of consensus exists regarding their therapeutic management particularly for recurrent disease. We present even low-risk dGIST may metastasize and require long-term observation, and a long-term prognosis may be achieved by combining resection and imatinib therapy for liver metastases recurrence.
\end{abstract}

Introduction:

Gastrointestinal stromal tumors (GISTs) are the most common type of primary mesenchymal tumors to originate from the gastrointestinal tract. GIST occurs in 11-19.6 per million people. ${ }^{1,2)}$ Duodenal GISTs (dGISTs) represent only $4-5 \%$ of all GISTs, and are extremely rare. ${ }^{3,4)}$ So, there is lack of consensus regarding optimal treatment particularly for recurrent of dGISTs. Herein we report a case of liver metastasis 7 years after resection of low-risk dGIST.

Case presentation:

The patient was a 45-year-old woman with Graves-Basedow disease who was found to have anemia during follow-up. Physical examination was unremarkable, and laboratory values were within normal ranges. Upper gastrointestinal endoscopy revealed a submucosal duodenal tumor with partial erosion (Fig. 1a). A bowel biopsy was not diagnostic. Endoscopic ultrasound (EUS) showed a hypoechoic mass originating in the muscularis mucosa (Fig. 1b). Contrast-enhanced abdominal computed tomography (CT) revealed a 30mm hyper-vascular tumor in the duodenal bulb (Fig. 2). The preoperative diagnosis was submucosal duodenal tumor, and the patient underwent partial resection of the duodenal bulb with distal gastrectomy, followed by Roux-en-Y reconstruction. The resected specimen contained a $20 \times 12-\mathrm{mm}$ submucosal tumor (Fig. 3). Histopathological evaluation revealed a tumor comprised of spindle-shaped cells including 5 mitotic figures per 50 high-power fields (HPFs). Immunohistochemical evaluation indicated that the tumor cells were positive for c-Kit and CD34 expression (Fig. 4). The tumor was diagnosed as low-risk dGIST based on the Fletcher classification, Miettien classification and modified Fletcher classification (also known as the Joensuu classification). Postoperative follow-up was continued, and 7years later, CT revealed a $39 \mathrm{~mm}$ enhanced tumor in liver segment 4 (Fig. 5a). Positron emission tomography (PET) showed accumulation of 18F-fluorodeoxyglucose in the tumor with a maximum standardized uptake value (SUVmax) of 3.8 (Fig. 5b). The tumor was diagnosed as a metastatic liver tumor, and the patient underwent partial hepatectomy. Macroscopic findings showed a $3.9 \times 3.0 \times 3.5 \mathrm{~cm}$ tan and grey solid tumor (Fig. 6). Histological analysis showed spindle cells with mitoses with 6 mitoses/50 HPFs and a Ki67 value of 7\%. Immunohistochemical analysis revealed positive staining for c-Kit and CD34 (Fig. 7). The tumor was diagnosed as a liver metastasis 
from the dGIST. The patient has received oral imatinib $400 \mathrm{mg}$ daily and remains free of disease 4 years after the last surgery.

Discussion:

The first choice for treatment of resectable primary GIST is complete surgical resection. ${ }^{5)}$ Postoperative follow-up should be based on recurrence risk categories, such as those proposed by Fletcher et al. ${ }^{6}$, Miettinen et al. ${ }^{7}$ and Joesuu et $\mathrm{al}^{8}$. The Fletcher' classification is based on tumor size and mitotic rate, while the Miettinen classification and modified Fletcher classification (also known as the Joensuu classification) is further divided by site of occurrence. Even though GIST with a small tumor size or inconspicuous mitotic figures may show metastases. In the Miettinen classification, the rate of metastases in low risk GIST each location is reported to be $3.6 \%$ in the stomach, $4.3 \%$ in the small intestine, $8.3 \%$ in the duodenum, and $8.5 \%$ in the large intestine. In 2008, Joensuu et al. reported a Modified Fletcher classification that further stratified each mitosis into 3 groups and considered cases of tumor capsule destruction as high-risk groups for recurrence. Modified Fletcher classification has been reported to be useful for selects only high-risk groups from other risk groups ${ }^{9}$.

In the present case, the tumor in the duodenum was deemed to be low risk according to the Fletcher classification, Miettinen classification, and modified Fletcher classification. Even today, adjuvant chemotherapy with imatinib is not recommended for such low-risk tumors. In patients with intermediate-risk, low-risk, or ultra-low-risk GIST, follow-up by abdominal CT is recommended every 6 to 12 months for 5 years after surgery, and then annually up to 10 years after surgery. But as in this case, even low risk, dGIST has be able to recur.

A PubMed search using the key terms 'duodenal GIST' and 'liver metastasis' revealed 9 cases reports including our case $\mathrm{e}^{10)-17)}$. Three cases had synchronous liver metastasis and six cases had metachronous liver metastasis. The time to appearance of liver metastasis ranged from 3 months to 29 years. Only one low-risk case other than the present case has been reported; in that case, the liver metastasis appeared 29 years after resection of the dGIST. This suggests that long-term follow-up is necessary even in patients with low-risk dGIST.

Generally, the most common sites of recurrence or metastasis in GIST are the liver and peritoneum ${ }^{6}$. According to a report by DeMatteo et al. ${ }^{18)}, 63 \%$ of recurrences occur in the liver. Even for resectable metastatic liver tumors, the frequency of recurrence after resection is $80 \%$ to $90 \%$ and most occur within 2 years ${ }^{19)}$. Thus, GIST is difficult to cure with surgery alone, and imatinib treatment is the first choice for GIST recurrence. On the other hand, the usefulness of multidisciplinary treatment combining imatinib treatment and surgical resection or radiofrequency ablation therapy (RFA) for metastatic GIST has been reported ${ }^{20)-24)}$. Sato et $\mathrm{al}^{25)}$ reported continuous imatinib therapy appears to be important primarily for the prognostic improvement of patients with recurrent/metastatic GISTs and R0/R1 resection may have benefits when combined with imatinib therapy for patients with stable disease or disease responsive to imatinib therapy. In previous dGIST liver metastasis reports, although the long-term outcomes were few included, one case have survived 10 years after liver resection combination with imatinib therapy.

In the present case, the liver metastasis appeared 7 years after surgery and was completely resected and imatinib has administered. The patient has remained alive without recurrence for 4 years after liver resection, suggesting a good prognosis can be obtained by combining $\mathrm{R} 0$ resection with imatinib therapy.

Thus, this case report suggests that surgery is appropriate in patients who experience dGIST recurrence, if $\mathrm{R} 0$ resection and imatinib therapy is possible.

\section{Conclusion:}

Low-risk dGIST can metastasize relatively long after surgery. However, an excellent long-term prognosis may be able to be achieved by combining complete resection and imatinib therapy in patients for liver metastases recurrence. 
Author contributions

M.I. wrote the manuscript. M.I. and M.S. designed the study. A.W., R.K., R.K., H.S., I.O., K.M.,M.I.,K.T., S.S.,T.T., proofread the manuscript.

References:

1. Goettsch W. G, et al. Incidence of gastrointestinal stromal tumours is underestimated: results of a nationwide study. Eur J Cancer.2005; 41: 2868-2872.

2. Nilsson B, et al. Gastrointestinal stromal tumors: the incidence, prevalence, clinical course, and prognostication in the preimatinib mesylate era-a population-based study in western Sweden. Cancer.2005;103: $821-829$.

3. Agaimy A, Wunsch PH. Gastrointestinal stromal tumors: a regular origin in the muscularis propria, but an extremely diverse gross presentation. Langenbecks Arch Surg. 2006; 391(4):322-329.

4. Winfield RD, Hochwald SN, Vogel SB, et al. Presentation and management of gastrointestinal stromal tumors of the duodenum. Am Surg. 2006;72:719-722.

5. Nishida T, Blay JY, Hirota S, Kitagawa Y, Kang YK. The standard diagnosis, treatment, and follow-up of gastrointestinal stromal tumors based on guidelines. Gastric Cancer.2016;19:3-14.

6. Fletcher CD, Berman JJ, Corless C, et al. Diagnosis of gastrointestinal stromal tumors: A consensus approach. Hum Pathol.2003;33:459-465.

7. Miettinen M, Lasota J. Gastrointestinal stromal tumors: pathology and prognosis at different sites. Semin Diag Pathol.2006;23(2):70-83.

8. Joensuu $\mathrm{H}$ : Risk stratification of patients diagnosed with gastrointestinal stromal tumor. Hum Pathol $2008 ; 39: 1411-1419$.

9. Joesuu H, Vehtari A, Riihimali J, et al: Risk of reccurence of gastrointestinal stromal tumour after surgery: an analysis of pooled population-based cohorts. Lancet Oncol.2012;13(3):265-274.

10. Choi WH, Kim S, Hyung WJ, et al. Long-surviving patients with recurrent GIST after receiving cytoreductive surgery with imatinib therapy. Yonsei Med J. 2009;50(3):437-440.

11. Bhattacharya S, Choudhury AK, Ravi S, et al. Six years survival on imatinib with no disease progression after diagnosis of metastatic duodenal gastrointestinal stromal tumour: a case report. J Med Case Rep. $2008 ; 2: 110$.

12. Sakakura C, Hagiwara A, Soga K, et al. Long-term survival of a case with multiple liver metastases from duodenal gastrointestinal stromal tumor drastically reduced by the treatment with imatinib and hepatectomy. World J Gastroenterol. 2006;12(17):2793-2797.

13. Cameron S, Schaefer IM, Schwoerer H, et al. Ten Years of Treatment with $400 \mathrm{mg}$ Imatinib per Day in a Case of Advanced Gastrointestinal Stromal Tumor. Case Rep Oncol. 2011;4(3):505-511.

14. Liu J, Zhang C, Hong D, et al. Percutaneous microwave ablation liver partition and portal vein embolization for planned hepatectomy due to large gastrointestinal stromal tumor metastases: A case report. Medicine (Baltimore). 2017;96(42):e8271.

15. Hygum K, Wulff CN, Harslof T, et al. Hypercalcemia in metastatic GIST caused by systemic elevated calcitriol: a case report and review of the literature. BMC Cancer. 2015;15:788.

16. Stratopoulos C, Soonawalla Z, Piris J, et al. Hepatopancreatoduodenectomy for metastatic duodenal gastrointestinal stromal tumor. Hepatobiliary Pancreat Dis Int. 2006;5(1):147-150. 
17. Ginori A, Scaramuzzino F, Marsili S, et al. Late hepatic metastasis from a duodenal gastrointestinal stromal tumor (29 years after surgery): report of a case and review of the literature. Int J Surg Pathol. 2015;23(4):317-321.

18. DeMatteo RP, Lewis JJ, Leung D, et al. Two hundred gastrointestinal stromal tumors: recurrence patterns and prognostic factors for survival. Ann Surg. 2000;231(1):51-58.

19. Dematteo RP, Heinrich MC, El-Rifai WM, et al: Clinical management of gastrointestinal stromal tumors: before and after STI-571. Hum Pathol 33(5):466-477,2002.

20. Rubio-Casadevall J, Martinez-Trufero J, Garcia-Albeniz G, et al. Role of Surgery in Patients With Recurrent, Metastatic, or Unresectable Locally Advanced Gastrointestinal Stromal Tumors Sensitive to Imatinib: A Retrospective Analysis of the Spanish Group for Research on Sarcoma (GEIS) Ann Surg Oncol. 2015; 22: 2948-2957

21. Gronchi A, Fiore M, Miselli F, et al. Surgery of Residual Disease Following Molecular-Targeted Therapy With Imatinib Mesylate in Advanced/Metastatic GIST. Ann Surg. 2007; 245: 341-346

22. Zaydfudim V, Okuno SH, Que FG,et al. Role of Operative Therapy in Treatment of Metastatic Gastrointestinal Stromal Tumors. J Surg Res. 2012; 177(2): 248-254

23. Hakime A, Cesne AL, Deschamps F, et al. A Role for Adjuvant RFA in Managing Hepatic Metastases From Gastrointestinal Stromal Tumors (GIST) After Treatment With Targeted Systemic Therapy Using Kinase Inhibitors Cardiovasc Intervent Radiol. 2014; 37: 132-139

24. Vassos N, Agaimy A, Hohenberger W, et al. Management of Liver Metastases of Gastrointestinal Stromal Tumors (GIST). Ann Hepatol. 2015; 14: 531-539

25. Sato S, Tsujinaka T, Masuzawa T, et al. Role of metastasectomy for recurrent/metastatic gastrointestinal stromal tumors based on an analysis of the Kinki GIST registry. Surg Today. 2017; 47: 58-64.

Figure legends:

Fig. 1. a)Upper gastrointestinal endoscopy revealed a submucosal duodenal tumor with partial erosion. b) EUS showed a hypoechoic mass originating in the muscularis mucosa.

Fig. 2. Contrast-enhanced abdominal CT revealed a 30-mm hyper-vascular tumor in the duodenal bulb.

Fig. 3. The resected specimen contained a 20x12-mm submucosal tumor.

Fig. 4. Histopathological evaluation revealed a tumor comprised of spindle-shaped cells including 5 mitotic figures per $50 \mathrm{HPF}$. Immunohistochemical evaluation indicated that the tumor cells were positive for c-Kit and CD34 expression.

Fig. 5. a) CT revealed a 39-mm enhanced tumor in liver segment S4. b) PET showed accumulation of $18 \mathrm{~F}$-fluorodeoxyglucose in the tumor with a SUVmax of 3.8.

Fig. 6. Macroscopic findings showed a 3.9x3.0x3.5-mm tan and grey solid tumor.

Fig. 7. Histological analysis showed spindle cells with 6 mitoses/50 HPFs and a Ki67 value of 7\%. Immunohistochemical analysis revealed positive staining for c-Kit and CD34.

Consent:

Written informed consent was obtained from the patients for publication of this case series and any accompanying images. A copy of the written consent is available for review by the Editor-in-Chief of this journal.

Competing interests:

There are no potential conflicts of interest to disclose. 


\section{Hosted file}

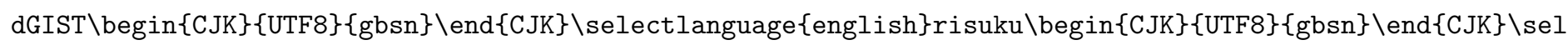
available at https://authorea.com/users/332527/articles/458940-a-liver-metastasis-7-yearsafter-resection-of-a-low-risk-duodenal-gastrointestinal-stromal-tumor

\section{Hosted file}

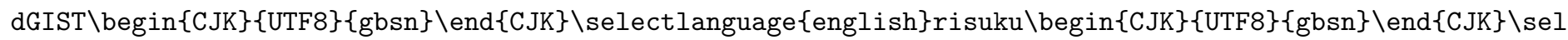
available at https://authorea.com/users/332527/articles/458940-a-liver-metastasis-7-yearsafter-resection-of-a-low-risk-duodenal-gastrointestinal-stromal-tumor

\section{Hosted file}

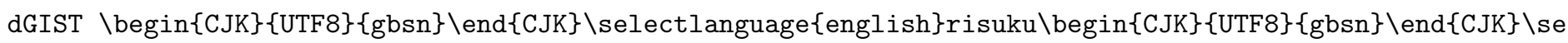
available at https://authorea.com/users/332527/articles/458940-a-liver-metastasis-7-yearsafter-resection-of-a-low-risk-duodenal-gastrointestinal-stromal-tumor

\section{Hosted file}

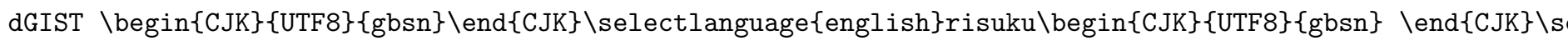
available at https://authorea.com/users/332527/articles/458940-a-liver-metastasis-7-yearsafter-resection-of-a-low-risk-duodenal-gastrointestinal-stromal-tumor

\section{Hosted file}

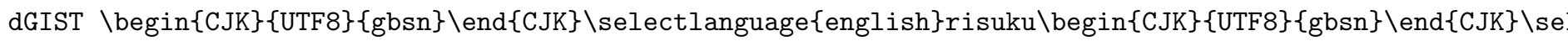
available at https://authorea.com/users/332527/articles/458940-a-liver-metastasis-7-yearsafter-resection-of-a-low-risk-duodenal-gastrointestinal-stromal-tumor

\section{Hosted file}

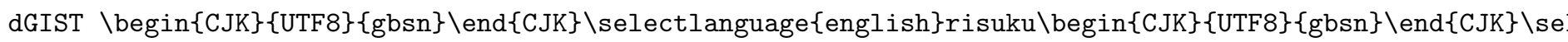
available at https://authorea.com/users/332527/articles/458940-a-liver-metastasis-7-yearsafter-resection-of-a-low-risk-duodenal-gastrointestinal-stromal-tumor

\section{Hosted file}

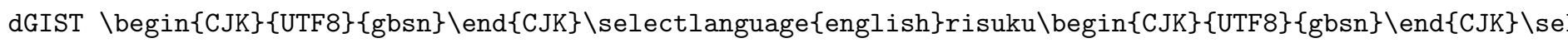
available at https://authorea.com/users/332527/articles/458940-a-liver-metastasis-7-yearsafter-resection-of-a-low-risk-duodenal-gastrointestinal-stromal-tumor 